\title{
Pré-natal de alto risco em um serviço de referência: perfil sociodemográfico e clínico
}

High-risk prenatal care in a reference service: sociodemographic and clinical profile

Pré-natal de alto riesgo en un servicio de referencia: perfil sociodemográfico y clínico

Daniele Socorro de Brito Souza Paiva ${ }^{1,2 *}$, Heliana Helena de Moura Nunes ${ }^{1,2}$, Sônia Fátima da Silva Moreira $^{3}$, Maria Goreth Silva Ferreira ${ }^{2}$

\section{RESUMO}

Objetivo: Descrever o perfil sociodemográfico e clínico de gestantes de alto risco. Métodos: Estudo transversal, retrospectivo e descritivo com abordagem quantitativa, elaborado a partir de coleta e análise de dados obtidos em prontuários de gestantes atendidas no pré-natal de alto risco da Fundação Santa Casa de Misericórdia do Pará no período de janeiro a junho de 2016. Resultados: A análise dos 536 prontuários incluídos na pesquisa demonstrou que $79,8 \%$ das gestantes estão nas faixas etárias abrangentes dos 15 aos 34 anos, $48,3 \%$ convivem em união estável, $78 \%$ são pardas, $48,3 \%$ possuem o ensino médio, $36 \%$ são do lar, 44,4\% são procedentes de Belém, $81 \%$ possuem antecedentes mórbidos familiares e 53,9\% antecedentes mórbidos pessoais. Em relação ao histórico obstétrico, 32,1\% são primigestas, $65,9 \%$ não tem antecedentes de abortamento e 90,1\% estavam com idade gestacional inferior a 32 semanas na primeira consulta médica. As três patologias mais prevalentes encontradas nesta pesquisa foram a hipertensão arterial (15,3\%), malformações fetais $(9,5 \%)$ e diabetes $(7,3 \%)$. Conclusões: Considerando a importância da identificação e intervenção dos fatores de risco gestacional para a redução da morbimortalidade materna e neonatal, é fundamental que pesquisas periódicas sejam realizadas para a identificação dos agravos mais prevalentes, subsidiando o planejamento de estratégias das políticas públicas. Neste estudo, as patologias mais frequentes foram a hipertensão arterial, malformações fetais e diabetes.

Palavras-chave: Gravidez de Alto Risco, Perfil de Saúde, Prevalência.

\begin{abstract}
Purpose: To describe the sociodemographic and clinical profile of high-risk pregnant women. Methods: A cross-sectional, retrospective and descriptive study with a quantitative approach, based on the collection and analysis of data obtained from the medical records of pregnant women attended at the high-risk prenatal of the Fundação Santa Casa de Misericórdia do Pará from January to June 2016. Results: The analysis of the 536 medical records included in the study showed that $79.8 \%$ of the pregnant women are in the 15 to 34 year old, $48.3 \%$ are stable marital status, $78 \%$ are mixed race, $48.3 \%$ have high school, $36 \%$ are housewife, $44.4 \%$ are from Belém, $81 \%$ have a family morbid antecedent and $53.9 \%$ a personal morbid antecedent. Regarding obstetric history, $32.1 \%$ are first pregnancy, $65.9 \%$ have no history of abortion and $90.1 \%$ were of gestational age less than 32 weeks at the first medical visit. The three most prevalent pathologies found in this study were
\end{abstract}

\footnotetext{
${ }^{1}$ Fundação Santa Casa de Misericórdia do Pará (FSCMP). * Email: dsbspaiva@gmail.com

2 Universidade do Estado do Pará (UEPA).

3 Universidade Federal do Pará (UFPA).
} 
hypertension (15.3\%), fetal malformations $(9.5 \%)$ and diabetes $(7.3 \%)$. Conclusiones: Considering the importance of the identification and intervention of gestational risk factors for the reduction of maternal and neonatal morbidity and mortality, it is fundamental that periodic researches be carried out to identify the most prevalent diseases, subsidizing the planning of public policy strategies. In this study, the most frequent pathologies were hypertension, fetal malformations and diabetes.

Keywords: High-Risk Pregnancy, Health Profile, Prevalence.

\section{RESUMEN}

Objetivo: Describir el perfil sociodemográfico y clínico de mujeres embarazadas de alto riesgo. Métodos: Estudio de corte transversal, retrospectivo y descriptivo con abordaje cuantitativo, elaborado a partir de recolección y análisis de datos obtenidos en prontuarios de mujeres embarazadas atendidas en el prenatal de alto riesgo de la Fundação Santa Casa de Misericórdia do Pará en el período de enero a junio 2016. El análisis de los 536 prontuarios incluidos en la investigación demostró que el 79,8\% de las gestantes están en las franjas etarias integrales de los 15 a los 34 años, el 48,3\% conviven en unión estable, el $78 \%$ son pardas, $48,3 \%$ poseen la enseñanza media, el $36 \%$ son del hogar, el $44,4 \%$ son procedentes de Belém, $81 \%$ poseen antecedentes mórbidos familiares y $53,9 \%$ antecedentes mórbidos personales. En cuanto al histórico obstétrico, el $32,1 \%$ son primigenias, el $65,9 \%$ no tiene antecedentes de aborto y el $90,1 \%$ estaban con edad gestacional inferior a 32 semanas en la primera consulta médica. Las tres patologías más prevalentes encontradas en esta investigación fueron la hipertensión arterial (15,3\%), malformaciones fetales $(9,5 \%)$ y diabetes (7,3\%). Conclusiones: Considerando la importancia de la identificación e intervención de los factores de riesgo gestacional para la reducción de la morbimortalidad materna y neonatal, es fundamental que se realizan investigaciones periódicas para identificar los agravios más prevalentes, subsidiando la planificación de estrategias de las políticas públicas. En este estudio, las patologías más frecuentes fueron la hipertensión arterial, las malformaciones fetales y la diabetes.

Palabras clave: Embarazo de Alto Riesgo, Perfil De Salud, Prevalencia.

\section{INTRODUÇÃO}

A gestação é um fenômeno fisiológico, com alterações físicas, sociais e emocionais. Porém, situações de risco podem estar presentes durante a gravidez e resultarem em desfechos desfavoráveis. Assim sendo, é fundamental que a avaliação dos fatores de risco gestacional seja permanente e sempre que possível devem ser investigados durante os períodos pré-concepcional, concepcional, gestacional, partal e puerperal (BRASIL, 2012).

O pré-natal, quando realizado com qualidade, pode melhorar os indicadores de saúde, tais como a morbimortalidade materna e neonatal, pois possibilita a identificação e intervenção das situações de risco de uma gravidez (BRASIL, 2013a).

A portaria no 1.020 / 2013 do Ministério da Saúde (MS) instituiu as diretrizes para a atenção à saúde na gestação de alto risco e define gestação, parto e puerpério de risco quando doenças preexistentes ou intercorrências que surgem na gravidez, parto ou puerpério podem comprometer o estado de saúde das gestantes. Estas situações de risco podem ser decorrentes de fatores orgânicos, socioeconômicos e demográficos (BRASIL, 2013b).

O objetivo do pré-natal nas gestações de alto risco é reduzir o risco que o binômio materno-fetal estão expostos ou reduzir suas prováveis consequências adversas, interferindo no percurso de gravidezes com maior probabilidade de complicações (BRASIL, 2012). 
A mortalidade materna é um problema de saúde pública não só nacional como também mundial, diante disso, foi definido como o quinto dos oito Objetivos de Desenvolvimento do Milênio (ODM) da Organização das Nações Unidas (ONU) melhorar a saúde das gestantes, com a meta de reduzir em 3/4 a Razão de Mortalidade Materna (RMM) até 2015. Em 1990 a RMM no Brasil era de 139 óbitos para cada 100 mil nascido vivos (NV) e a meta estabelecida foi de 35 mortes por 100 mil NV (COSTA, 2012).

Com o objetivo de melhorar a saúde das gestantes e, em especial, reduzir a mortalidade materna, o MS elaborou políticas públicas direcionadas às mulheres, tais como o Projeto Maternidade Segura criado em 1995, o Programa de Humanização do Pré-Natal e Nascimento (PHPN) em 2000, a Política Nacional de Atenção à Saúde da Mulher, em 2004, juntamente com o Pacto Nacional pela Redução da Mortalidade Materna e Neonatal, o Pacto pela Vida e a Política Nacional de Atenção Básica. Infelizmente nenhum desses programas foi totalmente implantado. E por fim, em 2011 foi lançado o Programa Rede Cegonha, que corresponde ao modelo de assistência de pré-natal atual que se baseia em uma rede de cuidados de saúde à gestante e à criança (MAMEDE e PRUDÊNCIO, 2015).

A Rede Cegonha foi instituída no âmbito do Sistema Único de Saúde (SUS) pela Portaria no 1.459 / 2011 do MS, devido a identificação da necessidade de adotar medidas destinadas a assegurar a melhoria do acesso, da cobertura e da qualidade do acompanhamento pré-natal, da assistência ao parto e puerpério e da assistência à criança (BRASIL, 2011).

Atualmente o Brasil tem a RMM de 62 casos a cada 100 mil NV, segundo o MS. Apesar de não ter alcançado a meta estabelecida pelos ODM da ONU, a taxa caiu pela metade desde 1990. Nos países desenvolvidos a RMM é inferior a 16 óbitos maternos por 100 mil NV (CARNEIRO, 2015).

A mortalidade materna é definida como a morte de uma gestante ou puérpera, independente da duração ou da localização da gravidez, decorrentes da própria gravidez ou agravada por ela ou por conduta relacionada a ela (COSTA, 2012; MONTENEGRO e REZENDE FILHO, 2014).

As mortes maternas podem ser divididas em causas obstétricas diretas ou indiretas. As obstétricas diretas decorrem de complicações que surgem durante a gravidez, parto ou puerpério (período de até 42 dias após o parto) ou de intervenções, omissões, tratamento incorreto ou cadeia de eventos resultantes de qualquer das causas mencionadas. As mortes obstétricas indiretas resultam de doenças preexistentes ou que se manifestam durante a gravidez e agravam-se pelos efeitos fisiológicos da gestação, como problemas circulatórios e respiratórios (BRASIL, 2013a; MONTENEGRO e REZENDE FILHO, 2014).

Existem, ainda, os óbitos maternos não obstétricos, que são decorrentes de causas acidentais ou incidentais não relacionadas com a gravidez ou com o seu manejo. Esses óbitos não são inseridos no cálculo da RMM (MONTENEGRO e REZENDE FILHO, 2014).

Os óbitos maternos diretos são passíveis de prevenção a depender da qualidade dos cuidados no prénatal, parto e puerpério. No Brasil correspondem a $66 \%$ das mortes maternas e estão relacionados a fatores socioeconômicos (DIAS et al., 2015).

Segundo Costa (2012) a identificação dos geradores da desigualdade de saúde, como exemplo raça e cor, escolaridade, classe social, renda, é de fundamental importância para as ações em saúde com impacto positivo na qualidade da assistência. Outra análise importante é a compreensão do quão vulnerável e exposta está a saúde das pessoas sob determinadas condições e correlacioná-la com os indicadores de saúde.

Conforme Campos et al. (2010), conhecer os problemas de saúde mais frequentes, suas causas e consequências é fundamental para o planejamento de ações em saúde. Os dados levantados são transformados em informação, que por sua vez, produz o conhecimento para subsidiar este planejamento.

Desta forma, conhecer o perfil sociodemográfico das pacientes e as principais patologias atendidas no Pré-natal de alto risco (PNAR) é de grande valia para o planejamento estratégico na saúde. Sendo assim o objetivo do presente estudo foi descrever o perfil sociodemográfico e clínico de gestantes de alto risco. 


\section{MÉTODOS}

Foi realizado um estudo transversal, retrospectivo e descritivo, com abordagem quantitativa, elaborado a partir de coleta e análise de dados obtidos em prontuários de gestantes atendidas no PNAR da Fundação Santa Casa de Misericórdia do Pará (FSCMP) no período de janeiro a junho de 2016.

A amostragem das gestantes foi por conveniência, pois foram selecionados os prontuários que estavam disponíveis nos arquivos da FSCMP no período da coleta de dados, sendo, desta forma, incluídos 536 das 710 pacientes atendidas no referido período.

Para viabilizar a pesquisa, foi elaborado um instrumento de coleta de dados e foram analisadas as seguintes variáveis categóricas (nominais e ordinais) e quantitativas, coletadas das fichas médica do PNAR e da folha de rosto: Identificação: idade, procedência, estado civil e raça; Inserção social: escolaridade e situação profissional; História clínica: antecedentes familiares e pessoais; História obstétrica: paridade, idade gestacional (na primeira consulta médica no serviço), patologia principal que motivou o encaminhamento, outras patologias. Na ficha médica do PNAR e na folha de rosto dos prontuários da FSCMP não constam os dados referentes ao nível socioeconômico das pacientes e por este motivo esta variável não foi pesquisada.

O projeto de pesquisa foi submetido ao Comitê de Ética em Pesquisa da FSCMP e aprovado com o número do parecer 66938317.3.0000.5171, sendo respeitados os princípios preconizados em pesquisas envolvendo seres humanos, de acordo com a Resolução 466/12 da Comissão Nacional de Ética em Pesquisa.

\section{RESULTADOS}

Os dados obtidos dos prontuários das gestantes do PNAR foram analisados com base em suas frequências absolutas e relativas.

A análise dos 536 prontuários incluídos na pesquisa demonstrou que as idades das gestantes do PNAR da FSCMP no período pesquisado variam dos 11 aos 45 anos, com leve predomínio dos 30 aos 34 anos. A maioria convivem em união estável, são pardas, possuem ou estão cursando o ensino médio, são do lar e residem fora de Belém (Tabela $\mathbf{1}$ ).

TABELA 1 - Características sociodemográficas de gestantes do PNAR na FSCMP

\begin{tabular}{|c|c|c|}
\hline IDADE & n (536) & $\%$ \\
\hline $11-14$ anos & 14 & $2,6 \%$ \\
\hline $15-19$ anos & 50 & $9,3 \%$ \\
\hline 20-24 anos & 116 & $21,6 \%$ \\
\hline 25-29 anos & 125 & $23,3 \%$ \\
\hline 30-34 anos & 137 & $25,6 \%$ \\
\hline 35-39 anos & 64 & $12,0 \%$ \\
\hline$\geq 40$ anos & 30 & $5,6 \%$ \\
\hline \multicolumn{3}{|c|}{ ESTADO CIVIL } \\
\hline Sem registro & 31 & $5,8 \%$ \\
\hline Solteira & 128 & $23,9 \%$ \\
\hline Casada & 114 & $21,3 \%$ \\
\hline União estável & 259 & $48,3 \%$ \\
\hline Divorciada & 4 & $0,7 \%$ \\
\hline \multicolumn{3}{|l|}{ RAÇA } \\
\hline Sem registro & 51 & $9,5 \%$ \\
\hline Branca & 44 & $8,2 \%$ \\
\hline Parda & 418 & $78,0 \%$ \\
\hline Negra & 22 & $4,1 \%$ \\
\hline Amarela & 1 & $0,2 \%$ \\
\hline
\end{tabular}




\begin{tabular}{llrr}
\hline & ESCOLARIDADE & & \\
\hline Sem registro & & 60 & $11,2 \%$ \\
Analfabeta & & 1 & $0,2 \%$ \\
Ensino Fundamental & & 128 & $23,9 \%$ \\
Ensino Médio & & 259 & $48,3 \%$ \\
Ensino Superior & OCUPAÇÃO & 88 & $16,4 \%$ \\
\hline & & & \\
\hline Sem registro & & 193 & $36,0 \%$ \\
Estudante & & 28 & $5,2 \%$ \\
Do lar & PROCEDÊNCIA & 193 & $36,0 \%$ \\
Atividade remunerada & & 122 & $22,8 \%$ \\
\hline & & \multicolumn{3}{c}{} \\
\hline Sem registro & & 5 & $0,9 \%$ \\
Belém & & 238 & $44,4 \%$ \\
Ananindeua & & 115 & $21,5 \%$ \\
Outros municípios & & 178 & $33,2 \%$ \\
\hline
\end{tabular}

Fonte: Resultados da pesquisa, 2018.

$\mathrm{Na}$ tabela 2, observamos que a maioria das gestantes possuem antecedentes mórbidos familiares (81\%), sendo que $41,2 \%$ apresentam dois antecedentes. A hipertensão arterial é o antecedente familiar mais frequente, seguido de diabetes e a associação dessas duas patologias está frequente em $68,7 \%$ dos casos com dois antecedentes presentes.

TABELA 2 - Antecedentes mórbidos familiares das gestantes do PNAR na FSCMP.

\begin{tabular}{lcc}
\hline ANTECEDENTES MÓRBIDOS FAMILIARES (AMF) & $\mathbf{n}(\mathbf{5 3 6 )}$ & $\%$ \\
\hline Sem registro & 19 & $3,5 \%$ \\
Ausente & 83 & $15,5 \%$ \\
Presente & 434 & $81,0 \%$ \\
\hline NÚMERO DE AMF & $\mathbf{n} \mathbf{( 4 3 4 )}$ & $\%$ \\
\hline Presença de um AMF & 176 & $40,6 \%$ \\
Presença de dois AMF & 179 & $41,2 \%$ \\
Presença de três AMF & 64 & $14,7 \%$ \\
Presença de quatro AMF & 15 & $3,5 \%$ \\
\hline PATOLOGIA & $\mathbf{n}$ & $\%$ \\
\hline Hipertensão arterial & 343 & $79,0 \%$ \\
Diabetes mellitus & 248 & $57,0 \%$ \\
Gemelaridade & 132 & $30,5 \%$ \\
Malformações & 35 & $8,1 \%$ \\
Outros & 17 & $3,9 \%$ \\
\hline
\end{tabular}

Fonte: Resultados da pesquisa, 2018. 
As gestantes com antecedentes mórbidos pessoais correspondem a $53,9 \%$ das pesquisadas, sendo mais frequente apenas uma patologia como antecedente, destes o histórico de infecção urinária é o mais prevalente, seguida de hipertensão arterial e diabetes (Tabela 3). A associação de hipertensão arterial e diabetes também é a mais habitual (17\%) quando na presença de dois antecedentes.

TABELA 3 - Antecedentes mórbidos pessoais das gestantes do PNAR na FSCMP.

\begin{tabular}{|c|c|c|}
\hline ANTECEDENTES MÓRBIDOS PESSOAIS (AMP) & n (536) & $\%$ \\
\hline Sem registro & 13 & $2,4 \%$ \\
\hline Ausente & 234 & $43,7 \%$ \\
\hline Presente & 289 & $53,9 \%$ \\
\hline NÚMERO DE AMP & n (289) & $\%$ \\
\hline Presença de um AMP & 214 & $74,1 \%$ \\
\hline Presença de dois AMP & 53 & $18,3 \%$ \\
\hline Presença de três AMP & 21 & $7,3 \%$ \\
\hline Presença de quatro AMP & 1 & $0,3 \%$ \\
\hline PATOLOGIA & $\mathbf{n}$ & $\%$ \\
\hline Infecção urinária & 125 & $43,3 \%$ \\
\hline Hipertensão arterial & 53 & $18,3 \%$ \\
\hline Diabetes mellitus & 30 & $10,4 \%$ \\
\hline Tabagismo & 26 & $9,0 \%$ \\
\hline Asma & 23 & $8,0 \%$ \\
\hline Etilismo & 22 & $7,6 \%$ \\
\hline Lúpus eritematoso sistêmico & 16 & $5,5 \%$ \\
\hline Doenças hematológicas & 16 & $5,5 \%$ \\
\hline Epilepsia & 13 & $4,5 \%$ \\
\hline Nefropatias & 11 & $3,8 \%$ \\
\hline Tireoidopatias & 10 & $3,5 \%$ \\
\hline Cardiopatias & 9 & $3,1 \%$ \\
\hline Patologias uterinas & 8 & $2,8 \%$ \\
\hline Hepatite B & 7 & $2,4 \%$ \\
\hline Drogas ilícitas & 5 & $1,7 \%$ \\
\hline Neuropatias & 4 & $1,4 \%$ \\
\hline Doenças reumatológicas & 3 & $1,0 \%$ \\
\hline Outras & 12 & $4,2 \%$ \\
\hline
\end{tabular}

Fonte: Resultados da pesquisa, 2018. 
A tabela 4 expõe o antecedente obstétrico das gestantes: $32,1 \%$ são primigestas; $44,8 \%$ são nulíparas; $65,9 \%$ não têm antecedentes de abortamento; $89,2 \%$ não têm mau passado obstétrico com história de natimorto ou neomorto; e a idade gestacional durante a primeira consulta médica no PNAR tem uma discreta predominância entre 16 e 19 semanas e 6 dias.

TABELA 4 - História obstétrica das gestantes do PNAR na FSCMP.

\begin{tabular}{|c|c|c|}
\hline \multicolumn{3}{|c|}{ ANTECEDENTES OBSTÉTRICOS } \\
\hline Gestação & n (536) & $\%$ \\
\hline Uma & 172 & $32,1 \%$ \\
\hline Duas & 146 & $27,2 \%$ \\
\hline Três & 107 & $20,0 \%$ \\
\hline Quatro ou mais & 111 & $20,7 \%$ \\
\hline Paridade & n (536) & $\%$ \\
\hline Nenhuma & 240 & $44,8 \%$ \\
\hline Uma & 158 & $29,5 \%$ \\
\hline Duas & 82 & $15,3 \%$ \\
\hline Três & 33 & $6,1 \%$ \\
\hline Quatro ou mais & 23 & $4,3 \%$ \\
\hline Abortamento & n (536) & $\%$ \\
\hline Nenhum & 353 & $65,9 \%$ \\
\hline Um & 126 & $23,5 \%$ \\
\hline Dois ou mais & 57 & $10,6 \%$ \\
\hline Natimorto / Neomorto & n (296) & $\%$ \\
\hline Ausente & 264 & 89,2 \\
\hline Presente & 32 & 10,8 \\
\hline Idade Gestacional & n (536) & $\%$ \\
\hline 6 semanas - 11 semanas e 6 dias & 80 & $15,0 \%$ \\
\hline 12 semanas - 15 semanas e 6 dias & 75 & $14,0 \%$ \\
\hline 16 semanas - 19 semanas e 6 dias & 100 & $18,6 \%$ \\
\hline 20 semanas - 23 semanas e 6 dias & 82 & $15,3 \%$ \\
\hline 24 semanas - 27 semanas e 6 dias & 75 & $14,0 \%$ \\
\hline 28 semanas - 31 semanas e 6 dias & 71 & $13,2 \%$ \\
\hline 32 semanas - 36 semanas e 6 dias & 46 & $8,6 \%$ \\
\hline A partir de 37 semanas & 7 & $1,3 \%$ \\
\hline
\end{tabular}

Fonte: Resultados da pesquisa, 2018. 
A presença de apenas uma patologia como indicação de encaminhamento da gestante ao PNAR foi mais predominante, correspondendo a $87,5 \%$. A três principais patologias mais frequentes encontradas nesta pesquisa foram a hipertensão arterial, malformações fetais e diabetes (Tabela 5). Dos casos de hipertensão arterial, $64,6 \%$ correspondem a forma crônica da doença e $35,4 \%$ a pré-eclâmpsia. Contudo, acrescentando os 14 casos que a doença hipertensiva na gravidez foi relatada como comorbidade, ou seja, associada à outra patologia principal, esse prevalência sobe para 18,9\%.

TABELA 5 - Patologias que motivaram o encaminhamento ao PNAR na FSCMP.

\begin{tabular}{|c|c|c|}
\hline NÚMERO PATOLOGIAS & n (536) & $\%$ \\
\hline Presença de uma patologia & 469 & $87,5 \%$ \\
\hline Presença de duas patologias & 52 & $9,7 \%$ \\
\hline Presença de três patologias & 13 & $2,4 \%$ \\
\hline Presença de quatro patologias & 2 & $0,4 \%$ \\
\hline PATOLOGIA PRINCIPAL & n (536) & $\%$ \\
\hline Doença hipertensiva & 82 & $15,3 \%$ \\
\hline Hipertensão arterial crônica & 53 & $64,6 \%$ \\
\hline Pré-eclâmpsia & 29 & $35,4 \%$ \\
\hline Malformações fetais & 51 & $9,5 \%$ \\
\hline Sistema nervoso central & 27 & $53,0 \%$ \\
\hline Outros sistemas & 17 & $33,3 \%$ \\
\hline Múltiplas malformações & 7 & $13,7 \%$ \\
\hline Diabetes & 39 & $7,3 \%$ \\
\hline Diabetes Mellitus & 30 & $76,9 \%$ \\
\hline Diabetes Gestacional & 9 & $23,1 \%$ \\
\hline Toxoplasmose & 32 & $5,9 \%$ \\
\hline Gemelaridade & 31 & $5,8 \%$ \\
\hline Incompetência ístmo-cervical & 18 & $3,4 \%$ \\
\hline Abortamento recorrente & 17 & $3,2 \%$ \\
\hline Mau passado obstétrico & 17 & $3,2 \%$ \\
\hline Patologia uterina: miomatose uterina & 16 & $2,8 \%$ \\
\hline Lúpus eritematoso sistêmico & 15 & $2,8 \%$ \\
\hline $\begin{array}{l}\text { Outras patologias em gestação atual: } \\
\text { polidrâmnio, ClUR }\end{array}$ & 15 & $2,8 \%$ \\
\hline Cardiopatia materna & 14 & $2,6 \%$ \\
\hline Ameaça de abortamento & 14 & $2,6 \%$ \\
\hline Epilepsia & 13 & $2,4 \%$ \\
\hline Pós-gestação molar & 12 & $2,2 \%$ \\
\hline Estupro consensual & 12 & $2,2 \%$ \\
\hline Neuropatias & 12 & $2,2 \%$ \\
\hline Doenças hematológicas & 11 & $2,0 \%$ \\
\hline Sífilis & 10 & $1,9 \%$ \\
\hline Placenta prévia & 10 & $1,9 \%$ \\
\hline Outras doenças maternas & 9 & $1,7 \%$ \\
\hline Hepatite B & 9 & $1,7 \%$ \\
\hline Tireoidopatia & 8 & $1,5 \%$ \\
\hline Baixo risco & 8 & $1,5 \%$ \\
\hline
\end{tabular}




\begin{tabular}{lll} 
Asma & 7 & $1,3 \%$ \\
Doenças hepáticas & 6 & $1,1 \%$ \\
Patologias em gestação anterior & 6 & $1,1 \%$ \\
Nefropatias & 5 & $0,9 \%$ \\
Doenças reumatológicas & 4 & $0,7 \%$ \\
Câncer & 4 & $0,7 \%$ \\
Lesão intraepitelial de alto grau (LIEAG) & 4 & $0,7 \%$ \\
Outras doenças infeciosas: & & \\
citomegalovírus, HIV, zika vírus e & 4 & $0,7 \%$ \\
leishmaniose visceral & & \\
Patologias ovarianas & 4 & $0,7 \%$ \\
Psicopatias & 4 & $0,7 \%$ \\
Prematuridade habitual & 3 & $0,6 \%$ \\
Usuárias de drogas & 3 & $0,6 \%$ \\
Idade materna avançada & 3 & $0,6 \%$ \\
Pós-conização & 2 & $0,4 \%$ \\
Iteratividade & 2 & $0,4 \%$ \\
\hline
\end{tabular}

Fonte: Resultados da pesquisa, 2018.

\section{DISCUSSÃO}

Conhecer os fatores de alto risco das gestantes pode contribuir positivamente no planejamento das ações em saúde desse público alvo, com impactos reais e mais efetivos. A identificação e manejo clínico adequado das principais patologias corrobora para a melhoria dos indicadores de saúde, em especial, a mortalidade materna e perinatal.

A maioria das gestantes do PNAR está nas faixas etárias que compreendem dos 15 aos 34 anos (79,8\%), dados similares foram encontrados por Anjos et al. (2014) com 82,9\% dos 16 aos 35 anos e por Costa et al. (2016) onde $82 \%$ tinham idade entre 15 e 35 anos de idade. Em contrapartida, 20\% das gestante têm idades inferiores a 15 anos ou superiores a 35 anos, ou seja, com características maternas individuais consideradas mais propensas a complicações obstétricas e neonatais, em especial aos distúrbios hipertensivos. Gravena et al. (2013) identificaram mais prevalência de prematuridade, baixo peso ao nascer e baixo índice de Apgar no quinto minutos nas gestações em adolescentes e com idade materna igual ou superior a 35 anos.

Merece destaque a presença de gestantes com até 14 anos de idade, o que é considerado resultante de estupro de vulnerável, somando-se às grávidas de 15 a 19 anos temos $11,9 \%$ de gravidezes na infância e adolescência. No entanto este número provavelmente não reflete a real situação da nossa região, visto que nosso serviço não é a referência em pré-natal na adolescência. As gestantes adolescentes têm mais riscos maternos-fetais, com destaque para anemia, pré-eclâmpsia, hemorragia no pós-parto, prematuridade, infecções genito-urinárias, recém-nascido com baixo peso e óbito neonatal (BOUZAS et al., 2014).

A gravidez na adolescência normalmente ocorre de forma não planejada e segundo estudo de Fernandes et al. (2017) tem como causa e consequência a baixa escolaridade, uma vez que frequentemente resulta em abandono ou baixo desempenho escolar e assunção dos afazeres domésticos, além do mais, essas adolescentes são mais propensas a serem vítimas de violência doméstica. Todavia, a educação é um elemento fundamental na prevenção de gravidezes na adolescência.

Gravena et al. (2013) identificaram que o início tardio e um menor número de consultas de pré-natal são frequentes nas gestantes adolescentes, reforçando a relevância da sensibilização e motivação desse público alvo pela equipe de saúde. Para Dias et al. (2015) o desconhecimento dos riscos gestacionais e a baixa escolaridade podem também resultar em uma baixa adesão ao pré-natal, aumentando ainda mais os riscos maternos e perinatais. 
Ferraz e Bordignon (2012) pesquisaram o perfil da mortalidade materna no Brasil e constataram maior frequência de óbitos maternos na faixa etária dos 20 aos 29 anos $(41,8 \%)$, solteiras $(53,1 \%)$, pardas $(42,7 \%)$ e com baixa escolaridade (44,5\%). Identificaram também diferenças regionais do coeficiente de mortalidade materna, com a Região Norte apresentando o segundo maior coeficiente, perdendo apenas para a Região Nordeste. Segundo Costa (2012) essa diferença regional na mortalidade materna é resultante das desigualdades de acesso aos serviços de saúde e da qualidade dos mesmos.

O presente estudo detectou a prevalência de algumas condições com menores riscos gestacionais: quase $70 \%$ das gestantes apresentam relações estáveis (convivem em união estável ou são casadas) e não foi identificada uma baixa escolaridade de nossas gestantes, fatos esses que podem contribuir positivamente para os desfechos dessas gestações de alto risco.

Por outro lado, foi observado que a maioria das gestantes não exerce atividade remunerada, o que pode contribuir para condições socioeconômicas desfavoráves e consequentemente aumentar os riscos gestacionais. Além do mais, mais da metade das gestantes se consideram pardas, esses dados vão ao encontro dos dados do último censo do Instituto Brasileiro de Geografia e Estatística (IBGE), visto que a raça parda é a mais frequente no nosso Estado (69,5\%) (IBGE, 2011).

Fernandes (2014) em uma pesquisa com gestantes internadas na maternidade da FSCMP identificou que $48,2 \%$ das gestantes tinham entre 21 a 30 anos, $60,2 \%$ eram pardas, $75 \%$ situação conjugal de união estável, $34,7 \%$ tinham o ensino médio completo, 75,4 eram donas de casa. Resultados semelhantes com os encontrados no presente estudo, ratificando as características sociodemográficas das nossas gestantes.

Contudo, Costa et al. (2016) em um estudo do perfil das gestantes de alto risco em um município paranaense, identificaram que a maioria das gestantes eram brancas $(62,3 \%)$ e casadas $(52,5 \%)$, divergendo do nosso estudo e explicado pelas nossas diferenças étnica e culturais. Dado semelhante encontrado foi o ensino médio como a escolaridade mais frequente em ambos estudos (47,5\%).

Os antecedentes mórbidos familiares estão presentes em $81 \%$ das gestantes, em especial a hipertensão arterial e diabetes, isolados e associados. Resultados semelhantes foram encontrados por Costa et al. (2016) que identificaram a presença de antecedentes familiares em $88,5 \%$ da população estudada, sendo a hipertensão arterial a mais frequente $(63,9 \%)$, seguida de gemelaridade e diabetes. A presença de antecedentes familiares de hipertensão arterial e de diabetes em parentes de $1^{\circ}$ grau pode ser fator de risco para o desenvolvimento dessas patologias, em especial no ciclo gravídico-puerperal (BRASIL, 2012).

Outro fator de risco importante é a presença de antecedentes mórbidos pessoais, que estão presente na maioria das gestantes pesquisadas, com destaque para o histórico de infecção urinária, seguido por hipertensão arterial e diabetes. Na presença de dois antecedentes a associação de hipertensão arterial e diabetes também é a mais habitual no histórico pessoal. Costa et al. (2016) identificaram em sua pesquisa que $52,5 \%$ das gestantes relataram a infecção urinária como antecedente pessoal, seguido de cirurgia pélvica prévia e hipertensão arterial sistêmica.

A presença de patologias na gravidez, como infecção urinária, hipertensão arterial, diabetes, entre outras, são fatores de risco para a prematuridade, aumentando a morbimortalidade perinatal, além do mais a hipertensão arterial crônica é um fator de risco para pré-eclãmpsia e diabetes e da mesma forma o diabetes também é um fator de risco para pré-eclâmpsia (ACOG, 2013; CUNNINGHAM et al., 2016; KAHHALE et al., 2018).

Quanto ao histórico obstétrico das gestantes, dados semelhantes foram encontrados por Fernandes (2014), que em um estudo em nossa maternidade identificou que $40,2 \%$ eram primigestas, $33,7 \%$ nulíparas e $65,8 \%$ não referiam abortamento. No entanto, em nosso estudo, chama a atenção ao fato de que se as primigestas fossem desconsideradas da análise, o histórico de abortamento está presente em $50,2 \%$ das gestantes e foi ainda observado a presença de mau passado obstétrico com história de natimorto ou neomorto em $10,8 \%$ das pacientes com história de parto anterior. 
Em relação a idade gestacional no momento da primeira consulta médica no PNAR, foi observado uma predominância e frequências equivalentes entre si dos grupos com menos de 32 semanas gestacionais, esses dados ressaltam a possibilidade dos fatores de riscos estarem presentes antes da gravidez ou de surgirem no decorrer da mesma. O que reforça a necessidade de um pré-natal de qualidade para a identificação correta desses fatores de risco e para o encaminhamento oportuno para um serviço de referência (BRASIL, 2012).

As três patologias mais frequentes encontradas neste estudo foram a hipertensão arterial, malformações fetais e diabetes. Merece destaque o fato de que juntas correspondem a $32,1 \%$ dos casos, o que mostra a pluralidade das patologias prevalentes na população estudada.

Em um município do Baixo Amazonas do Pará, dos 94 casos estudados de encaminhamento para um centro de referência em PNAR, a hipertensão arterial foi mais frequente $(40,4 \%)$, seguida por malformação fetal $(7,4 \%)$ e diabetes (7,4\%), ambas com 7 casos (ANJOS et al., 2014). Costa et al. (2016) também verificaram a hipertensão arterial $(24,6 \%)$ como principal motivo de acompanhamento para o PNAR em sua pesquisa com análise de 61 prontuários.

Botelho et al. (2014) identificaram que a hipertensão arterial é a principal causa de morte materna no Estado do Pará, com destaque para a pré-eclâmpsia e suas formas clínicas (causa direta), e em menor frequência hipertensão arterial pré-existente à gravidez (causa indireta). Além do mais, esta morte materna é mais frequente no puerpério, o que reforça a necessidade de assistência integral e de qualidade à saúde da gestante em todas as etapas do ciclo gravídico-puerperal.

O manejo clínico adequado da hipertensão arterial é fundamental para a redução da mortalidade materna, visto que é a principal causa de óbito materno no Brasil (COSTA, 2012). Segundo o Painel de Monitoramento da Mortalidade Materna, em 2016, tivemos 1.288 óbitos maternos totais declarados, destes as causas obstétricas diretas foram responsáveis por $68 \%$, sendo a hipertensão arterial responsável por quase $20 \%$ desses. Say et al. (2014) identificaram que $73 \%$ dos óbitos maternos no mundo foram decorrentes de causas obstétricas diretas, porém a hemorragia, especialmente durante e após o parto, foi causa mais frequente (27\%), seguida da hipertensão arterial (14\%).

Em relação as malformações fetais, as mais prevalentes são do sistema nervoso central, com destaque para a ventriculomegalia / hidrocefalia, seguidos de anencefalia. Esta última é considerada $100 \%$ prevenível com o uso do ácido fólico no período periconcepcional, reafirmando a importância de medidas e orientações nesse período (CUNNINGHAM et al.,2016).

O diabetes foi a terceira patologia mais encontrada na pesquisa. No presente estudo o diabetes mellitus pré-gestacional representa $76,9 \%$ dos casos de diabetes, enquanto que o gestacional $23,1 \%$. Esses dados vão de encontro aos da literatura e podem ser decorrentes de subdiagnóstico de diabetes gestacional. $O$ diabetes pré-gestacional corresponde a $10 \%$ e o diabetes gestacional a $90 \%$ dos casos de diabetes na gestação (BRASIL, 2012). Weinert et al. (2010) identificaram em um ambulatório de diabetes e gestação que $84 \%$ dos casos correspondiam ao diabetes gestacional, $8 \%$ ao diabetes mellitus tipo $2,6 \%$ ao diabetes mellitus tipo 1 e $2 \%$ aos outros tipos.

O diabetes na gestação é a endocrinopatia mais prevalente e está relacionado a elevados índices de morbimortalidade perinatal. Nas gestações com diabetes gestacional as complicações mais frequentes são macrossomia fetal, polidrâmnio, trabalho de parto prematuro, amniorrexe prematura, hipoglicemia neonatal, síndrome do desconforto respiratório neonatal e óbito neonatal, enquanto que no diabetes pré-gestacional, além das mesmas complicações do gestacional, ocorrem riscos aumentados de abortamento e malformações fetais (ACOG, 2018; MCCANCE et al., 2018). Por esses motivos é importante o tratamento adequado dessa patologia no intuito de reduzir tais consequências.

As principais causas da morbimortalidade materna e perinatal são conhecidas, portanto, as estratégias das políticas públicas devem incidir nessas causas e é de fundamental importância uma assistência obstétrica de qualidade, abrangendo ações durante a gravidez, parto e puerpério. 


\section{CONCLUSÕES}

É relevante conhecer o perfil sociodemográfico-clínico das gestantes de alto risco para o planejamento estratégico das ações em saúde. Considerando a importância da identificação e intervenção dos fatores de risco gestacional para a redução da morbimortalidade materna e neonatal, é fundamental que pesquisas periódicas sejam realizadas para a identificação dos agravos mais prevalentes, subsidiando o planejamento de estratégias das políticas públicas. As patologias mais frequentes encontradas nesta pesquisa foram a hipertensão arterial, malformações fetais e diabetes.

\section{REFERÊNCIAS}

1. ANJOS JCS, PEREIRA RR, FERREIRA PRC et al. Perfil epidemiológico das gestantes atendidas em um centro de referência em pré-natal de alto risco. Revista Paraense de Medicina, 2014; 28(2): 23-33.

2. BOTELHO, NM, SILVA IFMM, TAVARES JR et al. Causas de morte materna no Estado do Pará, Brasil. Revista Brasileira de Ginecologia e Obstetetrícia, 2014; 36(7): 290-295.

3. BOUZAS ICS, CADER AS, LEAO L. Gravidez na adolescencia: uma revisão sistemática do impacto da idade materna nas complicacões clínicas obstétricas e neonatais na primeira fase da adolescência. Adolescência \& Saúde, 2014; 11(3): 7-21.

4. BRASIL. Atenção ao pré-natal de baixo risco. 1.ed. rev. Brasília: Ministério da Saúde; 2013a.

5. BRASIL. Gestação de alto risco: manual técnico. 5.ed. Brasília: Ministério da Saúde; 2012.

6. BRASIL. Ministério da Saúde. Portaria no 1.020, de 29 de maio de 2013. Brasília: Diário Oficial da União; $2013 b$.

7. BRASIL. Ministério da Saúde. Portaria no 1.459, de 24 de junho de 2011. Brasília: Diário Oficial da União; 2011.

8. BRASIL. Painel de monitoramento da mortalidade materna, 2016.

9. CAMPOS FCC, FARIA HP, SANTOS MA. Planejamento e avaliação das ações em saúde. 2.ed. Belo Horizonte: Nescon/UFMG, 2010; 110p.

10. CARNEIRO JD. Mortalidade materna cai no Brasil, mas não atingirá meta da ONU.

11. COSTA AM. Política de saúde integral da mulher e direitos sexuais e reprodutivos. In: GIOVANELLA, L. et. al. Políticas e Sistemas de Saúde no Brasil. 2.ed. rev. e amp. Rio de Janeiro: Editora FIOCRUZ, 2012.

12. COSTA LD, CURA CC, PERONDI AR et al. Perfil epidemiológico de gestantes de alto risco. Cogitare enferm., 2016: 21(2): 01-08.

13. CUNNINGHAM, FG, LEVENO KJ, BLOOM SL et al. Obstetrícia de Williams. 24 ed. Porto Alegre: AMGH, 2016.

14. DIAS JMG, OLIVEIRA APS, CIPOLOTTI R et al. Mortalidade Materna. Revista Médica de Minas Gerais, 2015; 25(2): 168-174.

15. FERRAZA L, BORDIGNONB M. Mortalidade materna no Brasil: uma realidade que precisa melhorar. Revista Baiana de Saúde Pública, 2012; 36(2): 527-538.

16. FERNANDES MMSM, SANTOS AG, ESTEVES MDS et al. Fatores de riscos associados à gravidez na adolescência. Revista de enfermagem da UFPI, 2017; 6(3): 53-58.

17. FERNANDES TLR. Perfil das gestantes atendidas em um hospital de referência. Dissertação (mestrado) - Universidade Federal do Pará. Programa de pós-graduação em enfermagem, Belém, 2014; 80p.

18. INSTITUTO BRASILEIRO DE GEOGRAFIA E ESTATíSTICA (IBGE). Censo demográfico 2010. Características da população e dos domicílios. Resultado do universo. Rio de Janeiro, 2011.

19. KAHHALE S, FRANCISCO, RPV, ZUGAIB M. Pré-Eclampsia. Revista de Medicina (São Paulo), 2018; 97(2): $226-34$.

20. MCCANCE DR, MARESH M, DACKS DA. A Practical Manual of Diabetes in Pregnancy. 2ed. U.S.A.: Wiley Backwell, 2018; 430p.

21. MAMEDE FV, PRUDÊNCIO PS. Contribuições de programas e políticas públicas para a melhoria as saúde materna. Revista Gaúcha de Enfermagem, 2015; 36(esp): 262-266.

22. MONTENEGRO CAB, REZENDE FILHO J. Rezende Obstetrícia fundamental. 13.ed. Rio de Janeiro: Guanabara Koogan, 2014; 1088 .

23. SAY L, CHOU D, GEMMILL A et al. Global causes of maternal death: a WHO systematic analysis. Lancet Glob Health, 2014; 2: 323333.

24. THE AMERICAN COLLEGE OF OBSTETRICIANS AND GYNECOLOGIST (ACOG ). Hypertension in pregnancy. 2013; 89p.

25. THE AMERICAN COLLEGE OF OBSTETRICIANS AND GYNECOLOGIST (ACOG). Gestational Diabetes Mellitus. Obstetrics \& Gynecology. ACOG Practice Bulletin, 2018; 131(2): e49-64.

26. WEINERT LS et al. Diabetes e gestação: perfil clínico e laboratorial em pré-natal de alto risco. Rev. HCPA, 2010; 30(4): 334-341. 